\title{
水质监测中的质量控制策略探讨
}

\author{
沈力 \\ 河南省南水北调中线渠首生态环境监测中心 \\ DOI:10.32629/eep.v3i7.887
}

\begin{abstract}
[摘 要] 水资源作为环境保护中的重要一项, 质量好坏直接关系到人们的身体健康, 水质监测工作能 够为环境治理提供最直接的数据资料, 促进水资源管理工作的改进与完善。随着人们环境意识的提高, 如何通过环境监测质量控制措施保证监测数据的代表性、准确性、精确性、可比性和完整性变得越 来越重要, 环境监测质量控制的重要性也越来越突出。鉴于此, 文章就水质监测中的质量控制策略进行 了探讨。
\end{abstract}

[关键词] 水资源; 环境保护; 水质环境监测; 质量控制

中图分类号：X853 文献标识码：A

\section{1 当前水环境监测的发展现状}

随着我国社会经济的不断发展, 对 水的需求量不断增加, 水资源的开发利 用给我国带来了巨大的压力, 同时, 工业 生产的不规范、环保意识的缺乏和水资 源污染的加剧, 水环境健康问题也越来 越受到人们的重视。目前, 我国水资源严 重短缺, 特别是西北地区水资源十分紧 缺, 需要依靠有效开展水环境监测工作 来获得相应的数据, 才能实现水资源的 合理利用。目前, 我国水环境监测技术已 经达到了大河、大湖等区域的覆盖面积, 同时在监测技术和手段日趋完善, 实现 了科学的技术规范, 特别是涉及监测点 布设、样品采集、运输和质量分析报告 等相关内容都达到了较高的水平。我国 水环境监测工作以辐射模式为基础, 即 以水环境监测中心为核心, 以监测断面 为抓手, 形成全国性的水资源监测网络, 能够满足不同地区的水环境监测需求。 同时采用定点采样和移动采样, 保证监 测数据的实时性和准确性 ${ }^{[1]}$ 。

\section{2 水质监测的意义}

水质监测主要是为了准确有效地测 定出水体中污染物的种类及其浓度。可 以看出, 不同水体其水质内部的种类和 浓度都在不断地变化, 专业监测人员需 要对水体内部的质量全面地进行评价。 主要的监测项目主要是由宏观指标和微

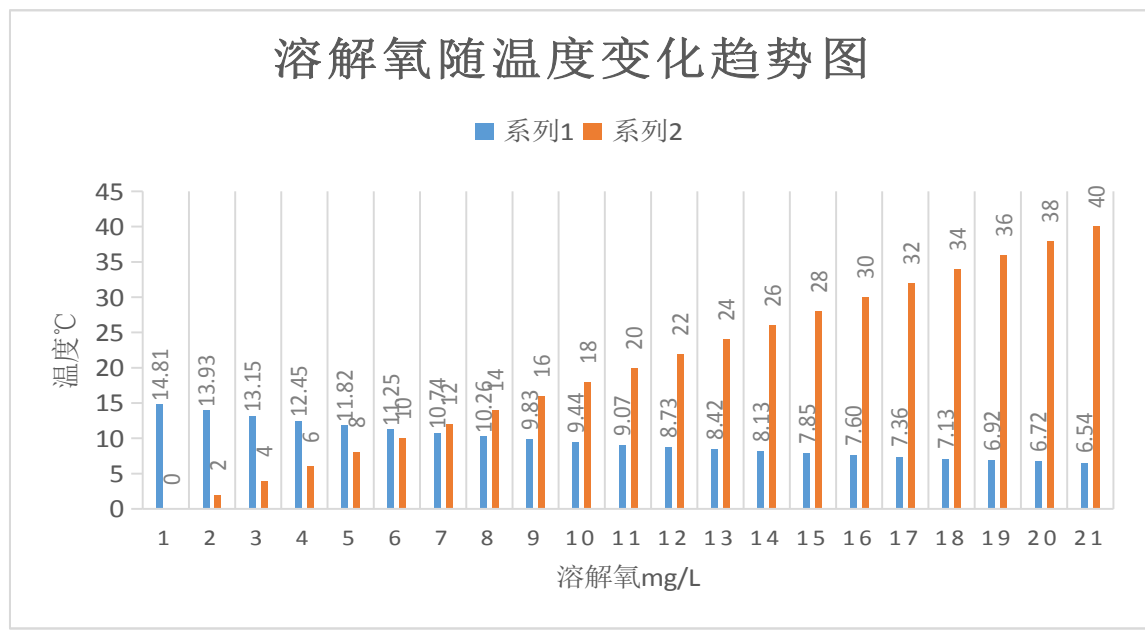

图 1 溶解氧随温度变化趋势图

观指标组成。其中, 宏观指标主要是悬浮 物、透明度、温度指标、pH指标、浊度、 电导率、溶解氧等, 微观指标主要包含水 中的有毒有害物质。因此, 只有通过有效 的水质监测结果才能够清楚了解水体内 部的具体情况, 这样才能够全面地指导 人们的生活用水。

\section{3 影响环境水质监测质量的} 因素

3. 1 采样过程的规范性。样品采集过 程规范与否, 直接关系到样品的代表性 和完整性, 是保证监测结果的准确性、精 密性、可比性的前提条件。采样断面和 采样点位的布设、采样频率和采样方法 的确定、采样容器的选取和洗涤, 以及样
品的预处理、保存和运输等, 都会对样品 的代表性和完整性产生影响。

3.2 实验室环境条件。实验室温度、 湿度、清洁度、采光、振动、噪声、辐 射等因素, 可通过影响分析仪器的精确 度, 对监测结果造成影响。以溶解氧为例, 在标准大气压、不同的温度下, 溶解氧也 随之变化, 如图:

3. 3监测方法的确定。在监测实验中, 监测方法并不是固定长期不变的, 因为 不同的监测方法会使监测结果大为不同, 检出限的差异也很明显。因此, 为了提升 监测结果的准确性, 在具体选择中应以 待测物种类和所用仪器的不同来选择合 适的方式。 
3. 4测试过程的把控。测试过程中, 实操人员对监测技术及有关规范标准的 掌握也必须非常熟练, 这在一定程度上 能提高监测结果的准确性。根据笔者在 实际工作中观察发现, 专业水准越高的 人员操作失误率越低, 他们在异常值的 处理和计算方面的能力也越强, 有效提 高了监测结果的质量。

3.5 分析仪器及试剂。分析仪器测量 范围、灵敏性等对监测结果有着直接的 影响, 若仪器的灵敏度低会降低测量低 浓度样品时的准确度, 且样品浓度越低, 误差越大。监测过程中使用的药品的纯 度不够则会使配置的试剂出现浓度误差, 降低监测结果的准确性, 配制试剂的水 的纯度不够也会产生同样的影响 ${ }^{[2]}$ 。

\section{4 环境水质监测质量控制策略}

4. 1水质监测环境的优化。在进行室 外现场监测时, 需充分评估外部环境是 否符合监测技术规范条件, 并及时对仪 器进行校准调整。水样的保存按照监测 技术要求, 保障科学的运输条件, 标明保 存条件及时间, 并及时送达化验室检验。 水质监测工作的开展需要一个良好的实 验室环境处于相对稳定的状态, 便于水 质监测工作的开展。同时, 需定期对设备 进行校正检验, 定期维护和保养设备, 提 高设备的使用寿命, 提高水质监测结果 的准确性。

4. 2 保证采集水样的代表性。首先, 应根据水样监测项目、监测目的来确定 水样采样容器、采样方法、采集量、保 存方式、采样频率、采样时间等。某些 项目的测定, 应在现场进行。其次, 要全 面考虑水质周边的环境, 之后再合理地 选择水质监测的位置。例如: 采集地表 水时应根据监测要求设置背景断面、采 样断面、控制断面、对照断面、入境断 面、出境断面等, 再在断面上设置采样垂 线数, 及各垂线上的采样点数。最后, 样 品是从各种水体及各类型水中取得的实 物证据和资料, 水样的采集是获得可靠
数据的前提条件 ${ }^{[3]}$ 。

4. 3 仪器设备运行质量控制。(1) 仪 器设备。水质分析过程中, 确保相关仪器 设备不妨碍最终监测结果的一个重要方 法就是校对所需的玻璃器血与监测仪器 等, 而校对的条件则是要根据国家已有 规范进行。除此之外, 在选择和使用仪器 过程中同样也必须要以国家的相关规范 为重要标准。(2) 标准物。就水质分析工 作而言, 标准物质扮演着十分关键的角 色。因为标准物质质量的好与否都会影 响到最终的监测结果的精确度。标准物 质选择过程中, 需要注意选择有证的标 准物质。若这一环节的难度较大, 那么还 可借助对比和验证来二次确定量值的精 确度与溯源性。而在使用标准物质时则 要注意其自身质量, 也就是按时核查, 避 免其质量出现问题, 进而影响到监测结 果。

4. 4 数据处理与综合评价质量保证。 (1) 数据处理。进行相关数据的处理工作 时, 主要进行对数据的记录整理工作和检 查等。其中对于数据的记录整理工作, 需 要工作人员要充分考虑到计量器具的精 密度以及准确性, 另外对于工作人员由于 失误, 可能会造成的读数误差也要进行注 意。同样, 对于数据的整理工作, 工作人员 也要根据一定的数据修约规则进行整理, 保证数据处理的有效性。(2) 综合评价质 量保证。进行综合性的分析评价工作时, 要充分对监测机构的主要技术水平进行 考察, 这也是进行水质监测工作的最终 环节, 是保证水质监测的质量控制与质 量保证的最终关卡。主要通过对监测数 据的分析利用, 得出综合评价。有效完成 对水环境质量定性结论的最终确定。并 且根据综合评论进行对水质下一步的防 治措施进行选择, 是改善水质的先决条 件, 可为水资源的可持续发展提供有效 根据。

4.5 提高水质监测人员的素质能力。 水质监测部门要加大资金的投入力度,
专门组织水质监测人员进行专业化和系 统性的培训, 使他们能够熟练掌握水质 监测技术和使用相关监测仪器, 让水质 监测人员自身的专业技能得到有效的 提升。同时, 加强对水质监测人员的技 能考核, 严格地按照规定, 非持证上岗 的监测人员不得进入监测部门工作; 定 期对监测人员的水质监测专业知识和 操作技能进行检验和考核, 制定出严格 的奖惩制度, 不仅可以保证监测人员基 本的专业技能可以全部地掌握通透, 而 且可以激发监测人员的工作积极性, 提 高监测工作的严谨度。现在是信息化、 数据化的时代, 水质监测工作中数据的 分析处理能力是监测人员需要提高的 重要部分。水质监测单位应该强化水质 数据分析和处理人员的能力, 培养计算 机软件的操作技术和数据分析的能力, 提升对实验数据的敏感程度。如此一来, 可以大大地提升监测水质结果的精确 度和科学性。

\section{5 结语}

总之, 为了保证用水质量、减少环境 水污染的发生, 水质监测工作至关重要。 水质监测通过对监测人员的选择、仪器 的选择以及如何保存和运输样本的方 式、实验室内外部比对来做到对水质监 测结果的质量控制。因此, 了解跟质控措 施的特点, 并根据自身实验室内部的具 体情况, 采样经济可行, 效果显著的质控 方式和质控措施, 是每个从事质控工作 的技术人员的研究方向。

\section{[参考文献]}

$[1]$ 吴金强.水质环境监测存在的问 题与优化方法初探 [J]. 清洗世 界,2019,35(12):44-45+47.

[2]刘阳.水质监测中的数据误差原 因分析及处理方法探讨 [J].水土保持应 用技术,2019,(06):42-43.

[3]黄贤伟.环境水质监测中的质量 控制影响要素与改进措施探讨 [ J ] 环境 与发展,2019,31(10):167-168. 